NUB1 has now been convincingly validated as a potent modulator of $\mathrm{mHTT}$ protein abundance and toxicity in several cell models and in Drosophila in vivo. But is NUB1 a viable therapeutic target for treating Huntington's disease? NUB1 itself is not an ideal target for smallmolecule drugs, as it is not known to possess receptor or enzymatic activity. However, NUB1 has been reported to be inducible by interferon- $\beta$ (IFN $\beta)^{10}$, an agent approved by the US Food and Drug Administration to treat the autoimmune disorder multiple sclerosis. Lu et al. ${ }^{1}$ explored this strategy of upregulating NUB1 by IFN $\beta$ treatment and observed an increase in NUB1, a decrease in mHTT and suppression of mHTT-mediated toxicity in Huntington's disease cell models. Moving these studies into rodent models of Huntington's disease in vivo will be an important next step toward uncovering the therapeutic potential of targeting NUB1 and the use of IFN $\beta$ as a treatment for Huntington's disease.

IFN $\beta$ is thought to slow disease progression in multiple sclerosis by modulating inflammation. Dysregulated immune signaling and inflammation have also been reported in Huntington's disease ${ }^{11}$. Although tests of IFN $\beta$ treatment to modulate inflammation in Huntington's disease have not been carried out, it is interesting to speculate that a potential mechanism of IFN $\beta$-induced suppression of inflammation in Huntington's disease might be through NUB1 or other IFN $\beta$ targets involved in protein homeostasis. IFNs have been reported to induce a response to oxidative stress and to upregulate key components of protein homeostasis machinery, such as ubiquitin and immunoproteasomes ${ }^{12}$, and immunoproteasomes have been reported to be induced in neurons from mouse models of Huntington's disease ${ }^{13}$. Future studies will help to more clearly define the possible link between protein degradation modifiers, such as NUB1, and inflammation in Huntington's disease.

Can targeting NUB1-mediated protein degradation be beneficial for treating other protein misfolding-related neurodegenerative diseases? NUB1 has been proposed to reduce, through regulation of glycogen synthase kinase- $3 \beta$ protein levels, phosphorylation and aggregation of the protein tau, which has been implicated in neurodegenerative disorders, such as Alzheimer's disease and other tauopathies ${ }^{14}$. NUB1 may also modulate proteasomal degradation of synphilin-1, a protein that interacts with $\alpha$-synuclein and is a major component of the Lewy bodies associated with dementia and Parkinson's disease ${ }^{15}$. Although more studies are needed to determine the implications of targeting NUB1 as a therapeutic strategy for neurodegenerative diseases, targeting proteasomal degradation of selected substrates has the potential to be broadly applicable to many diseases associated with expression and accumulation of toxic proteins.

\section{COMPETING FINANCIAL INTERESTS}

The authors declare no competing financial interests.

1. Lu, B. et al. Nat. Neurosci. 16, 562-570 (2013).

2. Yamamoto, A., Lucas, J.J. \& Hen, R. Cell 101, 57-66 (2000).

3. Kordasiewicz, H.B. et al. Neuron 74, 1031-1044 (2012).

4. Weiss, A. et al. Anal. Biochem. 395, 8-15 (2009).

5. Mitra, S., Tsvetkov, A.S. \& Finkbeiner, S. Autophagy 5 , 1037-1038 (2009)

6. Dragatsis, I., Levine, M.S.M. \& Zeitlin, S.S. Nat. Genet. 26, 300-306 (2000).

7. Kaltenbach, L.S. et al. PLoS Genet. 3, e82 (2007)

8. Liu, G. \& Xirodimas, D.P. Oncogene 29, 2252-2261 (2010).

9. Bae, B.-I. et al. Neuron 47, 29-41 (2005).

10. Kito, K. J. Biol. Chem. 276, 20603-20609 (2001).

11. Bouchard, J. et al. J. Neurosci. 32, 18259-18268 (2012).

12. Seifert, U. et al. Cell 142, 613-624 (2010).

13. Díaz-Hernández, M. et al. J. Neurosci. 23 11653-11661 (2003).

14. Richet, E. et al. Hum. Mol. Genet. 21, 5254-5267 (2012).

15. Tanji, K. et al. Am. J. Pathol. 169, 553-565 (2006).

\title{
Oligodendrocyte failure in ALS
}

Amyotrophic lateral sclerois (ALS) is a debilitating neurodegenerative disease that is characterized by the death of motor neurons and a progressive loss of all motor function. The motor neuron degeneration seen in ALS has also been intimately linked to glial cell dysfunction, which includes reactive gliosis, remyelination failure and alteration in glial metabolic support. Previous studies have documented that the glial precursor cells, which generate the myelinating cells of the CNS, known as $\mathrm{NG}_{2}^{+}$oligodendrocyte progenitor cells, exhibit greatly enhanced proliferation during the end stage of disease in the spinal cord of the SOD1(G93A) transgenic mouse model of ALS. However, the exact extent and timing of the oligodendrocyte abnormalities in ALS patients and the mouse model are unclear. In addition, the cause of the remyelination failure, and whether this is even important for disease pathology, remains unknown. On page 571 in this issue of Nature Neuroscience, Kang et al. show that $\mathrm{NG}^{+}$progenitor proliferation is enhanced before disease onset in this ALS mouse model.

$\mathrm{NG}_{2}{ }^{+}$oligodendrocyte progenitor cells are peppered throughout the gray matter of the spinal cord and are found in close proximity to neurons (as shown in this picture that depicts immunostaining of NG2 protein (green) and NeuN (red) in the ventral horn of mouse spinal cord). Kang et al. use genetic cell fate tracing techniques to


show an enhanced proliferation of $\mathrm{NG}^{+}$cells in the spinal cord gray matter of young, asymptomatic SOD1(G93A) mice. Surprisingly, however, these NG2 ${ }^{+}$cells fail to mature properly or sufficiently remyelinate spinal cord motor neurons. Although the $\mathrm{NG}^{+}$cells are able to differentiate into oligodendrocytes, the newly formed oligodendrocytes show morphological abnormalities, such as irregularly shaped cell processes and soma, and do not fully mature, as assessed by the reduction in remyelination and expression of myelin-forming proteins. The authors also find similar $\mathrm{NG}^{+}$and myelination defects from postmortem spinal cord and motor cortex samples taken from ALS patients. To determine whether these defects had any effect on disease onset or progression, the authors then returned to their mouse model of ALS, and genetically inactivated the ALS-linked mutant gene SOD1(G37R) specifically from NG2 ${ }^{+}$ glial progenitors. This delayed disease onset in the mice and increased their survival. These results suggest that preserving oligodendrocyte function may be a potential therapeutic target for combating this deadly disease.

Min Cho 